\title{
Change in Nutrition Strategy after Nutritional Support Team Activities for Cerebrovascular Disease Patients in the Intensive Care Unit
}

\author{
Jin Lee, Sung-Tae Kim, Won Hee Lee, Keun Soo Lee, Sung Hwa Paeng, Se Young Pyo, Young Gyun Jeong, \\ Moo Seong Kim, Yong Tae Jung
}

Department of Neurosurgery, School of Medicine, Inje University Busan Paik Hospital, Busan, Korea

Received: June 24, 2019

Accepted: July 8, 2019

\section{Corresponding Author:}

Sung-Tae Kim, M.D., Ph.D.

Department of Neurosurgery

Medicine, School of Medicine,

Inje University Busan paik

Hospital, Bok Ji Ro 75, Busan Jin-

$\mathrm{Ku}$, Busan, Korea

Tel:+82-51-890-6144

Fax:+82-51-898-4244

E-mail: kimst015@hanmail.net

\section{Objective}

This study was done to introduce a nutrition support strategy and analyze the effect of nutritional support team (NST) activities in a single-center intensive care unit.

\section{Methods}

Between January and June 2017, acute severe cerebrovascular disease (CVA) patients who admitted to the intensive care unit and referred to the NST, were enrolled and assigned to the NST group. Demographics, initial GCS score, needed calorie intake (NCI), type of nutrition, time of switching from parenteral nutrition (PN) to enteral nutrition (EN), NCI satisfaction rate, incidence of feeding intolerance (FI), and clinical outcomes were analyzed, and compared with those in the control group, retrospectively.

\section{Results}

There were 38 patients in the NST group and 39 in the control group. The initial GCS score was $7.03 \pm 2.19$ in the NST group and $6.82 \pm 2.19$ in the control group. The average NCI was 1557.9 $\mathrm{kcal}$ in the NST group and $1635.9 \mathrm{kcal}$ in the control group. In all patients, the start of PN was possible within 24 hours after admission. The EN start rate was slightly lower in the NST group. EN was initiated earlier in the NST group than in the control group. The NCI satisfaction rate at discharge was higher in the NST group. No statistically significant differences were observed in the rate of good clinical outcomes and incidence of FI between the two groups.

\section{Conclusion}

NST activity enabled the earlier start of EN and achievement of a high NCI satisfaction rate without increasing nutrition support related risks.

Keywords: Nutritional support; Cerebrovascular disorder; Intensive care unit 


\section{INTRODUCTION}

Appropriate nutritional support is key to the recovery of patients who are acutely ill or have undergone major surgery. Previous studies have focused to a certain degree on nutrition in the intensive care unit, and guidelines exist for the provision of nutritional support in such settings ${ }^{2,18}$. However, nutritional support strategies should be formulated based on each disease type. Cerebrovascular disease (CVA) is unique in that it is characterized by increased intracranial pressure, which may lead to gut-brain axis dysfunction $^{10,22)}$ and normal gastrointestinal tract function. Therefore, it is mandatory to consider such disease characteristics in the provision of nutritional support. However, few studies have focused on nutritional support for $\mathrm{CVA}^{11,21)}$.

Recently, our institute established a nutritional support team (NST) that includes neurosurgeons among others, for the active management of CVA patients. Significant changes have been observed in the nutritional support strategies employed after the performance of NST activities. The purpose of this study was to introduce our nutrition support strategy and the nutrition patterns used for CVA patients, and analyze the effect of NST activities in a single-center intensive care unit.

\section{MATERIALS AND METHODS}

\section{Study population}

This retrospective study was approved by the Institutional Review Board (Approval number, 19-0043).This analysis included patients with new-onset severe CVA (initial Glasgow Coma Scale [GCS] score 4 to 12), who were admitted to the intensive care unit of our institution. Acute ischemic stroke patients who underwent only medical treatment or intra-arterial thrombectomy were excluded. As NST activity was initiated only in 2015, the early years of patient data were not used to consider NST activity optimization. Between January and June 2017, patients who were referred to the NST were enrolled and assigned to the NST group (39 patients). The control group included patients with the same inclusion criteria, but who were enrolled from the period between January and June 2014 (38 patients).

\section{Nutrition support team and strategy}

The NST comprised neurosurgeons, general surgeons, dedicated nurses, pharmacists, and clinical dietitians. NST rounds were conducted twice a week, and the physicians alternately attended the round once a week. The needed calorie intake $(\mathrm{NCI})$ was calculated via the Harris-Benedict equation with the ideal weight ${ }^{20}$. There is no consistent definition for feeding intolerance (FI). Based on previous studies ${ }^{4,7,8,13-15)}$, we defined it as the presence of a gastric residual volume of $100 \mathrm{ml}>24 \mathrm{~h}$ on any day or gastrointestinal (GI) dysfunction such as diarrhea, abdominal distention, or signs of GI bleeding in the form of melena, hematochezia, hematemesis and bleeding via the L-tube. The team attempted to initiate PN within 24 hours from admission and then start EN, with $600 \mathrm{kcal}$ as the starting dose, as soon as possible after an intracranial pressure cri$\mathrm{sis}^{3,16)}$. EN and PN were provided in combination until EN covered about $70 \%$ of a patient's NCI. When FI was noticed, the team actively recommended GI motility drug use ${ }^{7}$, changed the EN formula, continuously implemented feeding techniques, and used other nutritional interventions. Once stable oral feeding was established, a 100\% NCI satisfaction rate was considered achieved.

\section{Data collection}

Data were collected equally from both the NST and control groups. Basic data on sex, age, weight, height, length of hospital stay and main diagnosis were collected. Information on the initial GCS score, NCI, type of nutrition in the form of PN and EN, time taken for $\mathrm{PN}$ and $\mathrm{EN}$ initiation, pattern of the increase in EN, NCI satisfaction rate, and clinical outcomes was analyzed. Data on FI and surgical interventions (craniotomy, stereotactic surgery, extra-ventricular drainage, neurointervention for aneurysm or arterio-venous malformation) were also collected. Clinical outcomes were measured using the modified Rankin scale (mRS).

\section{Statistical analysis}

Statistical analyses were performed using SPSS for Windows (version 25; SPSS, Chicago, IL, USA). In the statistical analyses, clinical outcomes were dichotomized into 'good' ( $\mathrm{mRS} 0$ to 4 ) and 'poor' (mRS 5 to 6). Factors related to nutrition were evaluated. Univariate analysis was performed with age, sex, initial GCS score, length of hospital stay, weight, height, body mass index (BMI), surgical intervention, NCI, PN start rate, EN start rate, EN-related parameters, NCI satisfaction rate on discharge, and clinical outcomes as factors using Pearson's chi-square test, t-test, Kruskal-Wallis test, and Fisher's exact test. Statistical significance was set at $\mathrm{p}<0.05$ for a $95 \%$ confidence interval.

\section{RESULTS}

The demographic characteristics of the patients are shown in Table 1 . There were 38 patients $(\mathrm{F} / \mathrm{M}=17 / 21$, mean age $63.37 \pm 13.59 \mathrm{y})$ in the NST group and 39 patients $(\mathrm{F} / \mathrm{M}=21 / 18$, mean age $65.51 \pm 16.78 \mathrm{y}$ ) in the control group, and the BMI values in the two groups were $22.48 \pm 3.35 \mathrm{~kg} / \mathrm{m}^{2}$ and $23.43 \pm 4.38$ $\mathrm{kg} / \mathrm{m}^{2}$, respectively. The initial GCS score was $7.03 \pm 2.19$ in the 
Table 1. Demographic characteristics of the nutrition support team group and control group

\begin{tabular}{lccc}
\hline & & Group & p-value \\
\cline { 2 - 3 } & NST (38) & Control (39) & $0.424^{*}$ \\
Sex, F/M & $17 / 21$ & $21 / 18$ & $0.540^{+}$ \\
Age (y) & $63.37 \pm 13.59$ & $65.51 \pm 16.78$ & $0.338^{+}$ \\
Weight $(\mathrm{kg})$ & $60.87 \pm 10.05$ & $63.67 \pm 14.86$ & $0.357^{+}$ \\
Height $(\mathrm{cm})$ & $164.42 \pm 7.94$ & $171.77 \pm 48.28$ & $0.291^{+}$ \\
BMI (kg/m $\left.{ }^{2}\right)$ & $22.48 \pm 3.35$ & $23.43 \pm 4.38$ & $0.681^{+}$ \\
GCS score & $7.03 \pm 2.19$ & $6.82 \pm 2.19$ & $0.715^{+}$ \\
Length of hospital stay & $33.16 \pm 28.77$ & $35.51 \pm 27.62$ & $0.192^{+}$ \\
Surgical intervention (\%) & $31(81.6)$ & $36(92.3)$ & \\
\hline
\end{tabular}

NST: nutritional support team; GCS: Glasgow coma scale; F: female; M: male; BMI: body mass index.

${ }^{*}$ Pearson's chi-square test.

${ }^{\dagger}$ t-test.

${ }^{\ddagger}$ Fisher's exact test.

NST group and $6.82 \pm 2.19$ in the control group. Thirty-one patients $(81.6 \%)$ in the NST group and $36(92.3 \%)$ in the control group received surgical intervention, and their lengths of hospital stay were $33.16 \pm 28.77$ and $35.51 \pm 27.62$ days, respectively. There was no statistically significant difference between the NST group and control group in each parameter.

Fig. 1 shows the nutrition flow of the two groups. Stroke was divided into three categories. Patients who underwent decompressive craniectomy due to large territory infarction were categorized as cerebral infarction patients, while those with spontaneous intracerebral hematoma (S-ICH) due to hypertensive causes or arteriovenous malformation ruptures were categorized as $\mathrm{S}-\mathrm{ICH}$ patients. Patients with spontaneous subarachnoid hemorrhage due to aneurysm rupture were categorized as cerebral aneurysm rupture patients. PN was initiated in all patients in both groups. EN was started in 29 patients in the NST group and 35 patients in the control group. Twenty-six of the 29 patients in the NST group and 15 of the 35 patients in the control group had an EN calorie increase. Six patients in both groups had FI. Twenty-six patients in the NST group and 24 in the control group were eventually discharged while under EN.

Table 2 shows the statistical findings of the feeding-related variables. There was no statistically significant difference in terms of the NCI between the two groups $(1,557.89 \pm 228.55$ vs $1,635.90 \pm 447.50 \mathrm{kcal}, \mathrm{p}=0.338)$. In all patients, $\mathrm{PN}$ could be initiated within 24 hours after admission. The EN start rate was lower in the NST group ( $76.3 \%$ vs $89.7 \%, \mathrm{p}=0.138)$. However, EN was initiated earlier in the NST group than in the control group (4.83 \pm 1.28 days vs $7.14 \pm 5.49$ days after admission, $p=0.02$ ). In addition, the interval between the start of EN and increase in calories was shorter in the NST group than the control group. ( $4.91 \pm 3.97$ vs $17.50 \pm 16.69$ days, $\mathrm{p}=0.028)$.

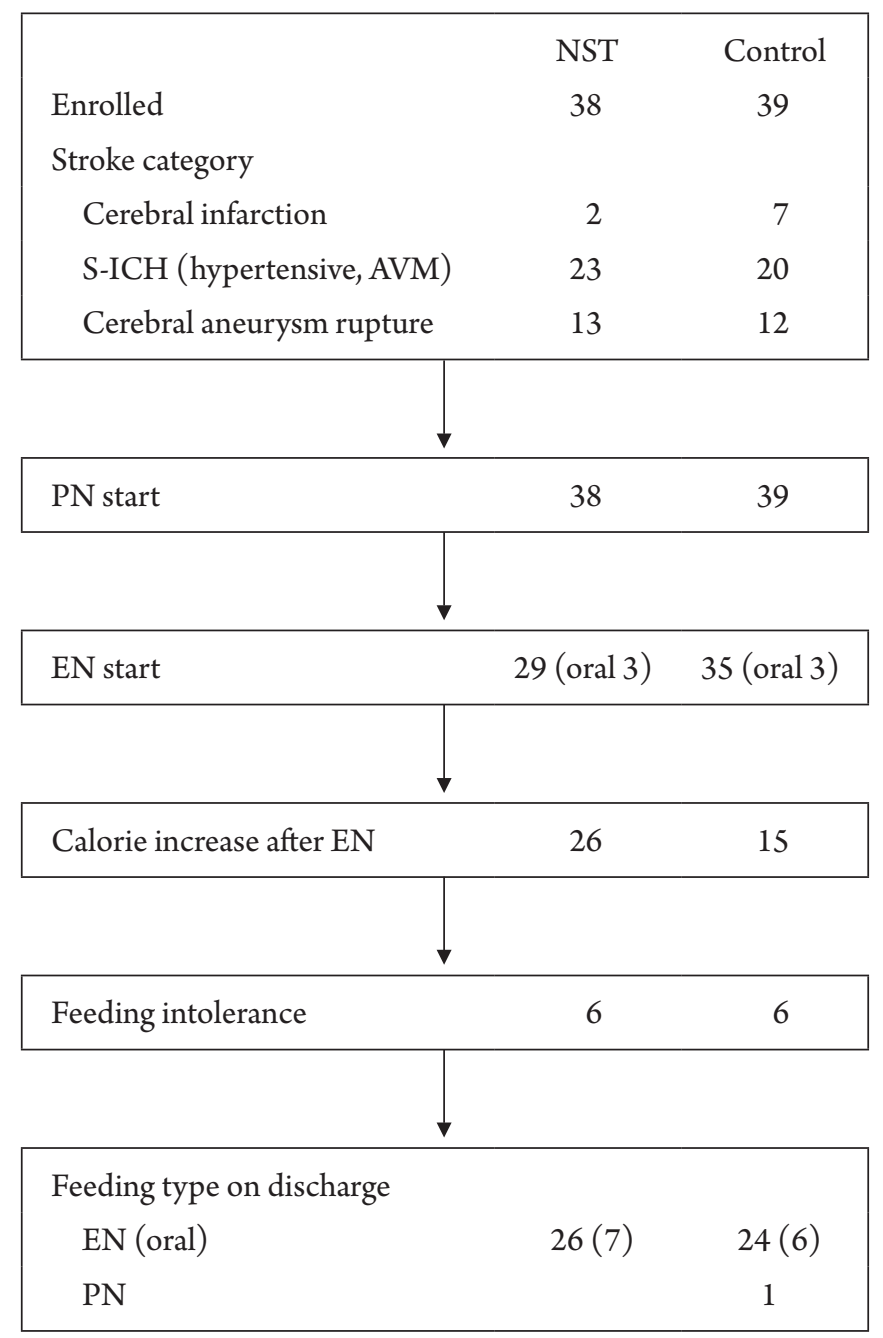

Fig. 1. Nutrition flow in the two groups of patients with cerebrovascular disease. NST, nutritional support team; S-ICH, spontaneous intracerebral hemorrhage, AVM, arteriovenous malformation; PN, parenteral nutrition; EN, enteral nutrition. 
Table 2. Statistically significant differences in the feeding-related variables between the nutritional support team group and control group

\begin{tabular}{|c|c|c|c|}
\hline & & & 1 \\
\hline & NST (38) & Control (39) & p-value \\
\hline Needed calorie intake & $1,557.89 \pm 228.55$ & $1,635.90 \pm 447.50$ & $0.338^{*}$ \\
\hline PN start (\%) & $38(100)$ & $39(100)$ & \\
\hline EN start (\%) & $29(76.3)$ & $35(89.7)$ & $0.138^{+}$ \\
\hline EN start day ${ }^{+}$ & $4.83 \pm 1.28$ & $7.14 \pm 5.49$ & $0.020^{*}$ \\
\hline EN start oral feeding & 3 & 3 & \\
\hline Calories at EN initiation & $685.7 \pm 269$ & $714.3 \pm 376$ & $0.736^{*}$ \\
\hline Patients with EN calorie increase $(\%)^{\ddagger}$ & $26(89.7)$ & $15(42.9)$ & $0.000^{+}$ \\
\hline Interval between EN start and calorie increase ${ }^{\S}$ & $4.91 \pm 3.97$ & $17.50 \pm 16.69$ & $0.028^{\|}$ \\
\hline Needed calorie intake satisfaction rate on discharge & $81.4 \% \pm 25.9$ & $56.1 \% \pm 28.4$ & $0.001^{*}$ \\
\hline Feeding intolerance $(\%)^{* *}$ & $6(20.7)$ & $6(17.1)$ & $0.757^{+}$ \\
\hline
\end{tabular}

NST: nutritional support team; EN: enteral nutrition; PN: parenteral nutrition.

*t-test.

${ }^{\dagger}$ Pearson's chi-square test.

${ }^{*}$ included in case of oral feeding.

${ }^{5}$ Number of patients in each group: $\mathrm{NST}=23$, Control $=12$.

"Kruskal-Wallis test.

INumber in each group: NST=26, Control $=25$.

**Number in each group: NST $=29$, Control $=35$.

Table 3. Clinical outcomes in the nutrition support team group and control group

\begin{tabular}{|c|c|c|c|}
\hline \multirow{2}{*}{$\mathrm{mRS}$ at 30 days } & \multicolumn{2}{|c|}{ Group } & \multirow{2}{*}{ p-value } \\
\hline & NST (38) & Control (39) & \\
\hline 1 & 1 & 1 & \\
\hline 2 & 1 & 0 & \\
\hline 3 & 3 & 2 & \\
\hline 4 & 9 & 9 & \\
\hline 5 & 13 & 13 & \\
\hline $6($ no EN $)$ & $11(9)$ & $14(4)$ & \\
\hline Good clinical outcomes $\mathrm{mRS} \leq 4$ & $14 / 38$ & $12 / 39$ & $0.573^{*}$ \\
\hline (\%) & -36.8 & -30.8 & \\
\hline
\end{tabular}

mRS: modified Rankin scale; NST: nutritional support team; EN: enteral nutrition; PN: parenteral nutrition.

*Pearson's chi-square test.

The EN starting calorie value was similar in both groups $(685.7 \pm 269 \mathrm{vs} 714.3 \pm 376 \mathrm{kcal}, \mathrm{p}=0.736)$. The NCI satisfaction rate on discharge was higher in the NST group than the control group $(81.4 \% \pm 25.9$ vs $56.1 \% \pm 28.4, \mathrm{p}=0.001)$. The incidence of FI was similar in two groups ( $20.7 \%$ vs $17.1 \%, \mathrm{p}=0.757)$.

Table 3 shows the differences in the clinical outcomes between the two groups. The NST group had a higher rate of good clinical outcomes $(\mathrm{mRS} \leq 4)$ on day 30 after admission $(36.8 \%, 14 \mathrm{pa}-$ tients) than the control group (30.8\%, 12 patients); however, statistical significance was not observed $(\mathrm{p}=0.573)$.

\section{DISCUSSION}

In our study, the NST and control groups had similar patient de- mographics and basic data, allowing for the easy comparison of the effects of the nutritional feeding activities. In both groups, PN could be initiated within 24 hours after admission. However, on average, EN was initiated faster in the NST group. Furthermore, the EN dose was initially increased later in the control group than the NST group, suggesting that the final NCI satisfaction rate on discharge may have been lower in the control group. Such differences can be attributed to the effects of the NST activities. The EN calorie increase in the control group was decided on by the neurosurgeon in charge. However, different neurosurgeons may have had different nutritional feeding strategies and may also have reacted to FI differently. In the NST group, owing to the presence of standardized strategies and goals, better clinical outcomes may have been possible. 
In the NST group, the percentage of patients in whom EN was started was lower than that in the control group. This could be attributed to the patients' overall condition on admission. EN was intentionally not initiated in patients who were diagnosed as having a low chance of neurological recovery or those in a coma. All such patients died. The rate of good clinical outcomes was higher in the NST group; however, the difference between the groups was not statistically significant. This could be because the study had a small sample size and patients' outcomes do not solely depend on nutrition. The clinical outcomes themselves should be further analyzed in future studies to establish if malnutrition can lead to the more rapid deterioration of health in $\mathrm{CVA}^{6,9)}$. Although statistically significant differences in terms of clinical outcomes were not observed in our retrospective study, faster EN initiation, high NCI satisfaction rate and low FI incidence rate in the NST group indicate that the administered NST activities led patients with severe CVA in the right nutritional strategic direction ${ }^{5,6,17)}$.

Despite the NST activities, the NST group patients' nutritional strategies did not adhere to those mentioned in previous guidelines for ICU patients ${ }^{2,18)}$. To reduce the rate of FI, length of ventilation and rate of infections, hypocaloric EN was provided and EN was initiated earlier in the early stages of ICU care in our NST group ${ }^{18)}$. However, it still took an average of 9 days or longer after admission for half the NCI to be reached, and about $80 \%$ of the NCI was eventually obtained via EN in the NST group at discharge. We may have taken FI too seriously compared to other models, leading to such results ${ }^{7,15}$. Compared to another study, the prevalence of FI (17-20\%) in our groups showed similar prevalence of FI in neurologic patients, which is lower than that noticed in cardiovascular, gastrointestinal, trauma and sepsis ICU patients ${ }^{7}$. The rate of FI seems to be influenced by a patient's condition or diagnostic category. The similar results, in terms of FI prevalence, observed in the patients with neurologic impairment may be due to the gut-brain axis; further research should focus on this aspect ${ }^{1}$. Even among stroke patients, large territory infarction patients and hemorrhagic stroke patients with slow clinical recovery may need different nutrition strategies compared to stroke patients who undergo intra-arterial thrombectomy with earlier clinical recovery.

The Harris-Benedict equation was used to estimate the energy expenditure in our patients. However, indirect calorimetry seems to be better for the estimation of energy expenditure in critically ill patients ${ }^{12}$; however, at present, its use is limited owing to the associated cost. In clinical practice, it is becoming increasingly common for diverse units comprising hospitalists and intensivists to work with physicians for treatment administration ${ }^{19)}$. The NST can be considered such a unit, as it strives to establish better treatment policies and achieve improved clinical outcomes. Furthermore, to enhance the role of the NST in the future, diverse nutritional strategies should be applied according to a patient's condition and diagnostic category.

A multicenter randomized controlled study Optimizing early enteral nutrition in severe stroke (OPENS) is being performed in China $^{21)}$. The results of that study and other similar studies must be considered in the formulation of further nutritional support strategies for the ICU.

A limitation of this study is its retrospective design. Therefore, the presence of selection bias and protocol deviations cannot be ruled out. Furthermore, the sample size was small; therefore, the results of the statistical analysis are limited. In addition, this study was single-center design and enrolled only people from Korea. For the establishment of nutrition-related guidelines for CVA patients, well-controlled prospective studies with a large sample size must be conducted.

\section{CONCLUSION}

NST activities enabled the earlier start of EN and the achievement of a high NCI satisfaction rate, without an increase in the risk of FI. Well-planned prospective studies are needed to reveal the clinical benefit of active nutritional support for CVA patients.

\section{NOTES}

\section{Conflict of interest}

No potential conflict of interest relevant to this article was reported.

\section{ACKNOWLEDGEMENTS}

This work was supported by 2018 Inje University Busan Paik Hospital research grant.

\section{REFERENCES}

1. Carabotti M, Scirocco A, Maselli MA, Severi C. The gut-brain axis: interactions between enteric microbiota, central and enteric nervous systems. Ann Gastroenterol 2015;28:203-209.

2. Carney N, Totten AM, O’Reilly C, Ullman JS, Hawryluk GW, Bell MJ, et al. Guidelines for the Management of Severe Traumatic Brain Injury, Fourth Edition. Neurosurgery 2017;80:6-15.

3. Cook AM, Peppard A, Magnuson B. Nutrition considerations in traumatic brain injury. Nutr Clin Pract 2008;23:608-620.

4. Deane AM, Fraser RJ, Chapman MJ. Prokinetic drugs for feed 
intolerance in critical illness: current and potential therapies. Crit Care Resusc 2009;11:132-143.

5. Giner M, Laviano A, Meguid MM, Gleason JR. In 1995 a correlation between malnutrition and poor outcome in critically ill patients still exists. Nutrition 1996;12:23-29.

6. Gramlich L, Kichian K, Pinilla J, Rodych NJ, Dhaliwal R, Heyland DK. Does enteral nutrition compared to parenteral nutrition result in better outcomes in critically ill adult patients? A systematic review of the literature. Nutrition 2004;20:843-848.

7. Gungabissoon U, Hacquoil K, Bains C, Irizarry M, Dukes G, Williamson R, et al. Prevalence, risk factors, clinical consequences, and treatment of enteral feed intolerance during critical illness. JPEN J Parenter Enteral Nutr 2015;39:441-448.

8. Mentec H, Dupont H, Bocchetti M, Cani P, Ponche F, Bleichner $\mathrm{G}$. Upper digestive intolerance during enteral nutrition in critically ill patients: frequency, risk factors, and complications. Crit Care Med 2001;29:1955-1961.

9. Oertel MF, Hauenschild A, Gruenschlaeger J, Mueller B, Scharbrodt W, Boeker DK. Parenteral and enteral nutrition in the management of neurosurgical patients in the intensive care unit. J Clin Neurosci 2009;16:1161-1167.

10. Ott L, Young B, Phillips R, McClain C, Adams L, Dempsey R, et al. Altered gastric emptying in the head-injured patient: relationship to feeding intolerance. J Neurosurg 1991;74:738-742.

11. Patejdl R, Kastner M, Kolbaske S, Wittstock M. Clinical nutrition and gastrointestinal dysfunction in critically ill stroke patients. Neurol Res 2017;39:959-964.

12. Picolo MF, Lago AF, Menegueti MG, Nicolini EA, Basile-Filho A, Nunes AA, et al. Harris-Benedict equation and resting energy expenditure estimates in critically ill ventilator patients. Am J Crit Care 2016;25:e21-29.

13. Reintam A, Parm P, Kitus R, Kern H, Starkopf J. Gastrointesti- nal symptoms in intensive care patients. Acta Anaesthesiol Scand 2009;53:318-324.

14. Reintam A, Parm P, Kitus R, Starkopf J, Kern H. Gastrointestinal failure score in critically ill patients: a prospective observational study. Crit Care 2008;12:R90.

15. Reintam Blaser A, Starkopf L, Deane AM, Poeze M, Starkopf J. Comparison of different definitions of feeding intolerance: A retrospective observational study. Clin Nutr 2015;34:956-961.

16. Ren G. Application of early nutrition support in neurosurgical coma patients. Indian J Surg 2015;77:1205-1208.

17. Schroeder D, Gillanders L, Mahr K, Hill GL. Effects of immediate postoperative enteral nutrition on body composition, muscle function, and wound healing. JPEN J Parenter Enteral Nutr 1991;15:376-383.

18. Singer P, Blaser AR, Berger MM, Alhazzani W, Calder PC, Casaer MP, et al. ESPEN guideline on clinical nutrition in the intensive care unit. Clin Nutr 2019;38:48-79.

19. Wise KR, Akopov VA, Williams BR, Ido MS, Leeper KV, Dressler DD. Hospitalists and intensivists in the medical ICU: a prospective observational study comparing mortality and length of stay between two staffing models. J Hosp Med 2012;7:183189.

20. Yatabe T. Strategies for optimal calorie administration in critically ill patients. J Intensive Care 2019;7:15.

21. Yuan F, Yang F, Zhang W, Jia Y, Ma Y, Qu Y, et al. Optimizing early enteral nutrition in severe stroke (OPENS): protocol for a multicentre randomized controlled trial. BMC Neurol 2019;19:24.

22. Zhu CS, Grandhi R, Patterson TT, Nicholson SE. A review of traumatic brain injury and the gut microbiome: insights into novel mechanisms of secondary brain injury and promising targets for neuroprotection. Brain Sci 2018;8:113. 